\title{
POSSIBLE OCCURRENCE OF A GENETIC BOTTLENECK IN DENGUE SEROTYPE 2 VIRUSES BETWEEN THE 1980 AND 1987 EPIDEMIC SEASONS IN BANGKOK, THAILAND
}

\author{
NOPPORN SITTISOMBUT, ANCHALEE SISTAYANARAIN, MARY JANE CARDOSA, MIKA SALMINEN, \\ SUPAPORN DAMRONGDACHAKUL, SIRIPEN KALAYANAROOJ, SUNTHAREE ROJANASUPHOT, \\ JIRAPORN SUPAWADEE, AND NIWAT MANEEKARN \\ Department of Microbiology, Faculty of Medicine, Chiang Mai University, Chiang Mai, Thailand; School of Pharmaceutical \\ Sciences, Universiti Sains Malaysia, Penang, Malaysia; Henry M. Jackson Foundation, Rockville, Maryland; \\ Department of Pediatrics, Children's Hospital, Ministry of Public Health, Bangkok, Thailand; \\ Virus Research Institute, Department of Medical Sciences, Ministry of Public Health, \\ Nonthaburi, Thailand
}

\begin{abstract}
Cocirculation of two genetic subtypes of dengue serotype 2 viruses was first observed in the 1980 epidemic season in Thailand. To further delineate the evolutionary history and the contribution of these subtypes to subsequent epidemics, we determined the envelope glycoprotein gene sequence of 20 dengue serotype 2 viruses isolated from infected patients during 1987 and compared them with those derived from earlier years. Subtype IIIa strains represented the majority (18 of 19) of dengue type 2 viruses derived from Bangkok metropolitan area, whereas all three strains from a province in the northeastern region belonged to subtype IIlb, indicating uneven local distribution of dengue subtypes within the same year. Three types of sequence variation were identified in both subtypes: substitutions that were unique to individual strains; substitutions that were shared among all subtype IIIa or IIIb viruses of both the 1980 and 1987 epidemics; and those that were shared only among all subtypes IIIa or IIlb viruses of the 1987 epidemic, but were absent from the corresponding subtypes of 1980 . While the first and second types of substitution were indicative of the most recent random mutations and previous mutations that had been fixed in virus populations, respectively, the third type suggested possible occurrence of a genetic bottleneck and subsequent expansion of one or a limited number of subtype IIIa strains in Bangkok between 1980 and 1987 . Immunoblot analysis of intracellular NS1 antigen with anti-NS1 monoclonal antibodies also revealed antigenic heterogeneity of the NS1 protein that correlated with the subdivision based on envelope protein variation.
\end{abstract}

Dengue viruses belong to the family Flaviviridae. Four serologic types have been detected, all of which had been associated with a wide range of illnesses in human, including undifferentiated fever, classical dengue fever, and dengue hemorrhagic fever (DHF) with/without unusual manifestations. ${ }^{1,2}$ A number of mosquito species can transmit dengue viruses from viremic subjects to others, but Aedes aegypti plays the major role in the cycle of transmission in urban settings. ${ }^{3.4}$ The geographic distribution of dengue viruses was paralleled with those of mosquito vectors involving many tropical and subtropical countries. ${ }^{5}$

An outbreak of DHF in Thailand was first recognized in Bangkok in 1958.6 Following the initial outbreak, dengue viruses spread to all parts of the country establishing an endemo-epidemic pattern. ${ }^{7}$ In Bangkok, concurrent transmission of multiple dengue serotypes was characteristic, ${ }^{8.9}$ but dengue serotype 2 appeared to be disproportionally associated with DHF ${ }^{8}$ It is not yet clear whether this observation reflects differences in virus-host interaction or higher virulence of dengue serotype 2 viruses.

While antigenic and sequence variations of dengue serotype 2 viruses have been well correlated with geographic separation at the global level, ${ }^{10-13}$ changes observed among viruses circulating within smaller localities and those that were related to temporal partitioning are less well studied. A longitudinal study using RNAse T1 oligonucleotide fingerprinting of 54 isolates derived from mosquitoes and patients during 1962-1986 in Thailand clearly revealed the heterogeneous nature of dengue type 2 viruses and also indicated slow and continual changes of a single population of viruses over time. ${ }^{14}$ However, examination of up to 10 serotype 2 viruses isolated in Bangkok in 1980 by fingerprint- ing and sequence analysis of the envelope and nsl genes revealed a high level of structural differences, allowing the subdivision of dengue type 2 viruses in Bangkok into two groups. ${ }^{15-17}$ This division was later confirmed by phylogenetic analysis of a larger dengue serotype 2 database; the two groups from Thailand were designated as subtypes IIIa and IIIb, respectively. ${ }^{13}$ Subtype IIIa viruses represented as many as $88 \%$ of all dengue type 2 viruses in this season (1980). ${ }^{15}$

Cocirculation of distinct genetic subtypes of dengue viruses is unlikely to be unique to Thailand. During the years 1986-1987, viruses belonging to subtypes IIIa and IIIb were identified among dengue type 2 viruses in Malaysia ${ }^{13}$ and two other subtypes were detected in Burkina Faso. ${ }^{12}$ However, the situation in Thailand appeared to be unusually complex as, in addition to the presence of two subtypes of dengue type 2; there was also evidences for three and two subtypes of dengue types 1 and 3, respectively, circulating within a period of 10 years. ${ }^{12.18}$ How these viral subtypes arise and interact with each other and with other coexisting serotypes is still unknown. To further delineate the genetic relationship and evolution of dengue virus serotypes, we determined the envelope gene sequence and heterogeneity of NS 1 antigens of dengue type 2 viruses isolated in Thailand during 1987 and compared them with previously published sequences. In 1987, the subtype IIIb viruses remained a minority in Bangkok; however, they might have flourished over the subtype IIIa in at least one province in the northeastern region of the country. Moreover, subtype IIIa viruses that had been isolated from various localities within the Bangkok metropolitan area throughout this epidemic year shared a novel base substitution, indicating that they may have passed 
TABLE 1

Characteristics of dengue type-2 viruses isolated from the Bangkok metropolitan area and two other provinces in 1987

\begin{tabular}{|c|c|c|c|c|c|c|c|c|}
\hline No. & Strain & Study code & $\begin{array}{c}\text { Date of } \\
\text { isolation } \\
\text { (1987) }\end{array}$ & $\begin{array}{l}\text { District of } \\
\text { Bangkok }\end{array}$ & $\begin{array}{c}\text { Patient age } \\
\text { (years) }\end{array}$ & $\begin{array}{l}\text { Serologic } \\
\text { response* }\end{array}$ & $\underset{\text { gradet }}{\text { DF/OHF }}$ & $\begin{array}{l}\text { GenBank } \\
\text { accession } \\
\text { no } / \text { Ref. }\end{array}$ \\
\hline 1 & D87-011 & TH87-011 & Jan & Smutprakan $\ddagger$ & 7 & $2^{\circ}$ & III & U34933 \\
\hline 2 & D87-167 & TH87-167 & Feb & Bangsue & 7 & $2^{\circ}$ & IV & U34943 \\
\hline 3 & D87-199 & TH87-199 & Mar & Smutprakan & 4 & $2^{\circ}$ & III & U34944 \\
\hline 4 & D87-279 & TH87-279 & Mar & Phayathai & 3 & $2^{\circ}$ & III & U34945 \\
\hline 5 & D87-300 & TH87-300 & Mar & Bangkhen & 5 & $2^{\circ}$ & IV & U34946 \\
\hline 6 & D87-499 & TH87-499 & May & Phayathai & 12 & $2^{\circ}$ & III & U34947 \\
\hline 7 & D87-642 & TH87-642 & Jun & Pratoomwan & 9 & $2^{\circ}$ & I & U34948 \\
\hline 8 & D87-704 & TH87-704 & Jun & Pratoomwan & 15 & $2^{\circ}$ & III & U34949 \\
\hline 9 & D87-738 & TH87-738 & Jun & Pratoomtani§ & 7 & $2^{\circ}$ & IV & U34950 \\
\hline 10 & D87-881 & TH87-881 & Jul & Bangkhen & 11 & $2^{\circ}$ & DF & U34951 \\
\hline 11 & D87-1040 & TH87-1040 & Aug & Promprab & 6 & $2^{\circ}$ & III & U34934 \\
\hline 12 & D87-1077 & TH87-1077 & Aug & Pratoomtani & 5 & $2^{\circ}$ & III & U34935 \\
\hline 13 & D87-1113 & TH87-1113 & Sep & Huaykwang & 3 & $2^{\circ}$ & II & U34936 \\
\hline 14 & D87-1121 & TH87-1121 & Sep & Bangkoknoi & 13 & $2^{\circ}$ & I & U34937 \\
\hline 15 & D87-1283 & TH87-1283 & Oct & Pratoomtani & 10 & $2^{\circ}$ & II & U34938 \\
\hline 16 & D87-1372 & TH87-1372 & Nov & Pratoomwan & 0.25 & $1^{\circ}$ & I & U34939 \\
\hline 17 & D87-1418 & TH87-1418 & Nov & Huaykwang & 10 & $2^{\circ}$ & III & U34940 \\
\hline 18 & D87-1421 & TH87-1421 & Nov & Yannawa & 0.3 & $1^{\circ}$ & IV & U34941 \\
\hline 19 & D87-1441 & TH87-1441 & Dec & Pratoomwan & 13 & $2^{\circ}$ & II & U34942 \\
\hline 20 & DST87-60 & TH87-060 & Aug & Kanchanaburiq & 7 & $2^{\circ}$ & IV & U34952 \\
\hline 21 & MK 42-86 & TH86-MK42 & Feb & Maha Sarakham & 2.5 & $2^{\circ}$ & DF & 31 \\
\hline 22 & MK 244-87 & TH87-MK244 & Jul & Maha Sarakham & 5 & $2^{\circ}$ & DHF & 31 \\
\hline 23 & MK 116-87 & TH87-MK 116 & May & Maha Sarakham & 5 & $2^{\circ}$ & DSS & 31 \\
\hline
\end{tabular}

$* 1^{\circ}=$ hemagglutination inhibition (HI) antibody titer against dengue antigens increases four-fold or higher by the end of a seven-day interval of illness and the convalescent titer is 1,280 or less. $2^{\circ}=\mathrm{HI}$ antibody titer against dengue antigens increases four-fold or higher and the convalescent titer is at least 2,560 .

+DF = dengue fever; patients were infected with dengue virus as revealed by a specific HI antibody test but did not have increased vascular permeability and thrombocytopenia; DHF = dengue hemorthagic fever; dengue-infected patients who manifested an increased vascular permeability and thrombocytopenia; grade I = fever, nonspecific constitutional symptoms, and a positive tourniquet test result: II = fever and spontaneous bleeding: III = fever, spontaneous bleeding. and circulatory failure: IV = fever, spontaneous bleeding. and profound shock with undetectable blood pressure and pulse; DSS = dengue shock syndrome; patients with grades III and IV DHF.

$\ddagger$ An adjacent province south of Bangkok.

$\$$ An adjacent province north of Bangkok.

$\$$ An adjacent province north of Bangkok.

1 A province in the northeastern part of Thailand.

through a genetic bottleneck during the seven-year period between 1980 and 1987. Genetic bottleneck refers to the phenomenon in which only one or a few, instead of all or the majority, of several members of cocirculating viruses actually give rise to descendant viruses in the later time period.

\section{MATERIALS AND METHODS}

Dengue viruses. Dengue serotype 2 viruses were isolated during 1987 from patients who were hospitalized at the Children's Hospital in Bangkok. Clinical diagnosis and grading of disease severity were according to the World Health Organization criteria. ${ }^{19}$ Briefly, this system classifies DHF grade $I$ as fever, nonspecific constitutional symptoms, and a positive tourniquet test result; grade II as fever and spontaneous bleeding; grade III as fever, spontaneous bleeding, and circulatory failure; and grade IV as fever, spontaneous bleeding, and profound shock with undetectable blood pressure and pulse. Viruses were isolated at the Department of Virology, Armed Forces Research Institute for Medical Sciences in Bangkok by intrathoracic inoculation of patients' sera into Toxorhynchites amboinensis and 4-5 passages in the C6/36 clone of Aedes albopictus mosquito cells. An indirect immunofluorescence assay was used for serologic classification. ${ }^{20}$ The following monoclonal antibodies were used: $4 \mathrm{G} 2$, anti-flaviviruses; $3 \mathrm{H} 5$, anti-dengue virus type 2 ; $1 F 1$, anti-dengue virus type $1 ; 10 \mathrm{C} 10$, anti-dengue virus type 3 ; and $1 \mathrm{H} 10$, anti-dengue virus type 4 (kindly provided by Drs. B. Innis, Department of Virology, Armed Forces Re- search Institute of Medical Sciences, Bangkok and D. Trent, Centers for Disease Control and Prevention, Fort Collins, CO). Pertinent clinical and epidemiologic information for these isolates is summarized in Table 1.

Virus culture and quantitation. Aedes albopictus cells $(\mathrm{C} 6 / 36)$ were seeded into $25-\mathrm{cm}^{2}$ tissue culture flasks at $5 \times$ $10^{5}$ cells per flask in Leibovitz 15 media containing $10 \%$ tryptose phosphate broth, $50 \mu \mathrm{g} / \mathrm{ml}$ of streptomycin sulfate, $50 \mathrm{U} / \mathrm{ml}$ of penicillin, and $10 \%$ fetal bovine serum. Cells were allowed to adhere overnight at $28^{\circ} \mathrm{C}$ after which the medium was replaced with $1 \mathrm{ml}$ of maintenance medium containing only $1 \%$ fetal bovine serum and two drops of infected culture supernatant or uninfected control medium were added to each flask. The virus was allowed to infect cells by rocking at room temperature overnight after which the medium was replaced with fresh maintenance medium and incubated in a stationary position. The cultures were examined under an inverted microscope daily and observed for cytopathic effect. Medium was changed when small syncytia were apparent and viral lysates were harvested when larger syncytia were observed, usually on the fifth or sixth day after inoculation. Quantitation of viruses by a four-step peroxidase-antiperoxidase method was performed on a monolayer of BHK-21 cells in 96-well plates and expressed as focus-forming units. ${ }^{21.22}$

Preparation of genomic RNA, amplification, and sequence analysis. Culture fluid of infected $\mathrm{C} 6 / 36$ cells was added to an equal volume of $0.1 \mathrm{M}$ Tris- $\mathrm{HCl}, \mathrm{pH} 8.0,0.1$ M EDTA, $0.2 \mathrm{M} \mathrm{NaCl}, 1 \%$ sodium dodecyl sulfate (SDS), 
Table 2

Oligonucleotide primers for amplification and nucleotide sequence analysis*

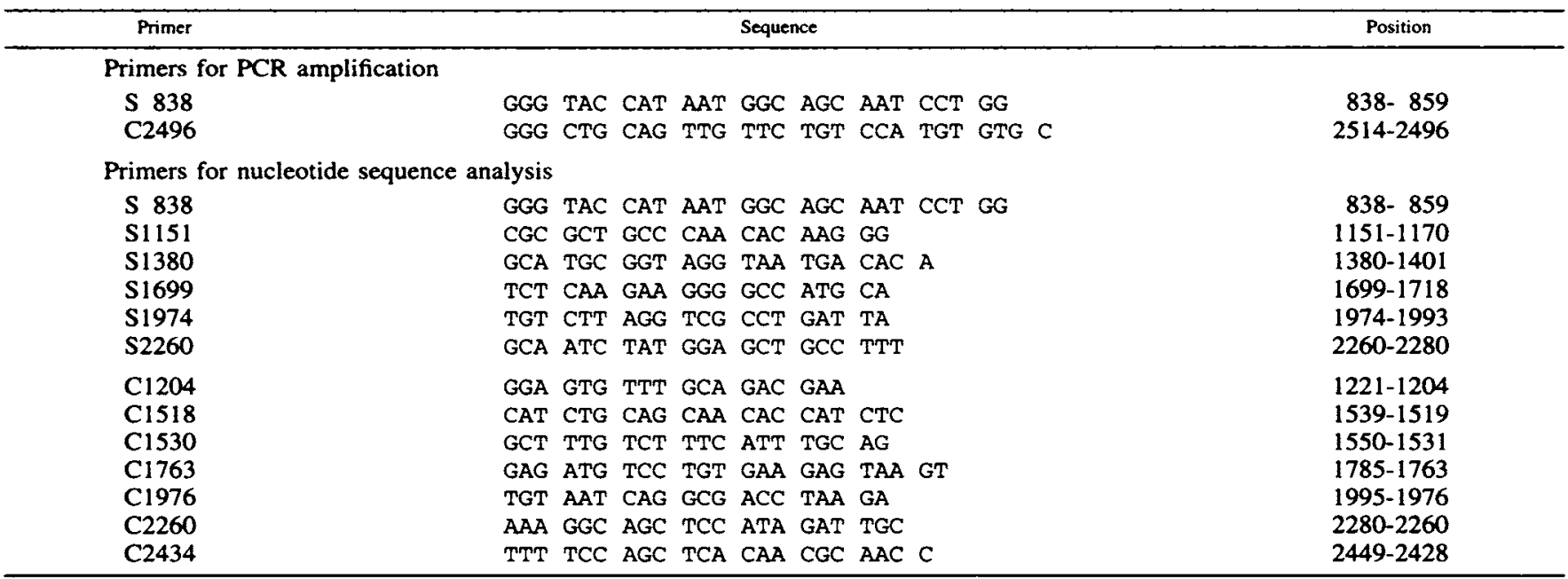

- PCR = polymerase chain reaction.

and $100 \mu \mathrm{g} / \mathrm{ml}$ of proteinase $\mathrm{K}$ and incubated at $56^{\circ} \mathrm{C}$ for $1.5 \mathrm{hr}$. Following extraction with phenol and chloroform, viral RNA was recovered by ethanol precipitation and resuspended in RNAse-free water. Genomic RNA derived from $4 \times 10^{4}$ focus forming units was heated to $70^{\circ} \mathrm{C}$ for 10 $\mathrm{min}$, chilled on ice, and reverse transcribed in $10 \mathrm{mM}$ Tris$\mathrm{HCl}, \mathrm{pH} 8.3,1.5 \mathrm{mM} \mathrm{MgCl}, 50 \mathrm{mM} \mathrm{KCl}, 0.001 \%$ gelatin, $10 \mathrm{mM}$ dithiothrietol, $0.5 \mathrm{mM}$ of each dNTP, 20 pmol of antisense primer, and $150 \mathrm{U}$ of reverse transcriptase (GIBCO/BRL, Gaithersburg, MD) at $45^{\circ} \mathrm{C}$ for $1 \mathrm{hr}$. The cDNA was amplified in 100 ul of $10 \mathrm{mM}$ Tris- $\mathrm{HCl}, \mathrm{pH} 8.3,1.5$ $\mathrm{mM} \mathrm{MgCl}{ }_{2}, 50 \mathrm{mM} \mathrm{KCl}, 0.001 \%$ gelatin, $0.1 \mathrm{mM}$ of each dNTP, 10 pmol of sense primer, and $2.5 \mathrm{U}$ of Taq DNA polymerase for 35 cycles of denaturation $\left(94^{\circ} \mathrm{C}\right.$ for $\left.1 \mathrm{~min}\right)$, annealing, and extension $\left(72^{\circ} \mathrm{C}\right.$ for $\left.5 \mathrm{~min}\right)$. Following separation on a $1 \%$ agarose gel, the 1,700-basepair DNA band was excised and purified. Approximately 50 fmol of polymerase chain reaction (PCR) products were used in the cycle sequencing reaction (GIBCO/BRL) with $5^{\prime}$ end-labeled primers according to the supplier's protocol. Base sequence was determined on both strands for strain D87-1421, which exhibited the highest sequence variation. The other 19 strains differed from each other at 0-29 positions; they were analyzed only on the noncoding strand in groups of 4-6 strains in parallel. Primers are listed in Table 2.

To examine the reliability of nucleotide sequence derived by cycle sequencing, the envelope gene of strain 16681, a laboratory-adapted dengue serotype 2 virus isolated from a fatal case of DHF in Thailand in $1964,{ }^{23}$ was also analyzed. When compared with previously published sequences, ${ }^{16.24} \mathrm{a}$ single base difference (C509 versus $U$ ) was detected on both cDNA strands of the products derived from two independent PCRs; the discrepancy most likely reflected a difference in the passage history of strain 16681 .

Phylogenetic analysis. The envelope gene sequences were aligned and the phylogenetic relationship were assessed with the fastDNAml program (Olsen G, University of Illinois, Urbana, IL and Matsuda H, Hagstrom R, Overbeek R, Argonne National Laboratory, Lemont, IL), which was de- rived from the DNAML program of the PHYLIP package.25 In addition, parsimony analysis was performed with 100 bootstrap replicates using DNAPARS and DNABOOT from PHYLIP. In the PHYLIP package of analytical softwares, the DNAML program uses the maximum likelihood procedure to determine the phylogenetic relationship amomg viruses whereas the DNAPARS program uses the principle of maximum parsimony. The DNABOOT program allows an evaluation of the significance of phylogenetic tree topology by bootstrapping. Strain SL 1050 (SL69-1050) was used as an outgroup because its bootstrap value with the whole group was only $37 \%$. In a previous study, strain SL69-1050 was placed differently in four equally parsimonious phylogenetic trees, ${ }^{13}$ indicating that this strain was not strongly associated with any particular dengue subtype.

Generation of anti-NS1 monoclonal antibodies. Mice (BALB/c) were infected with $0.5 \mathrm{ml}$ of infected culture fluid intraperitoneally. Three to six weeks later, the mice were immunized intravenously with $0.1 \mathrm{ml}$ of virus lysate and their spleens were removed after three days. Fusion of the spleen cells with Sp2/0 myeloma cells was performed according to Kohler and Milstein, ${ }^{26}$ and hybridomas were screened for dengue virus specific antibodies by a dot enzyme immunoassay described elsewhere. ${ }^{27}$ Supernatants from positive clones were tested by immunoblotting for NS1 specificity and recloned by limiting dilution at least twice. Dengue type 2 vinus strain 16681 as well as New Guinea C were used in separate fusions.

Preparation of cell lysate and immunoblot analysis. Culture fluids were removed and the infected $\mathrm{C} 6 / 36$ cell monolayers were washed once with phosphate-buffered sa-

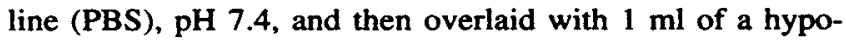
tonic buffer (20 mM HEPES, $5 \mathrm{mM} \mathrm{KCl}, 10 \mathrm{mM} \mathrm{MgCl}$, $0.05 \%$ Triton $\mathrm{X} 100$ ) and placed on a rocking platform for $5 \mathrm{~min}$. The monolayers were then resuspended, collected into a dounce homogenizer, and subjected to 20 strokes of the homogenizer. The lysates were then clarified by centrifugation at $1,500 \times g$ for $15 \mathrm{~min}$ at $4^{\circ} \mathrm{C}$ and the supernatants recovered were used as infected cell lysates. 


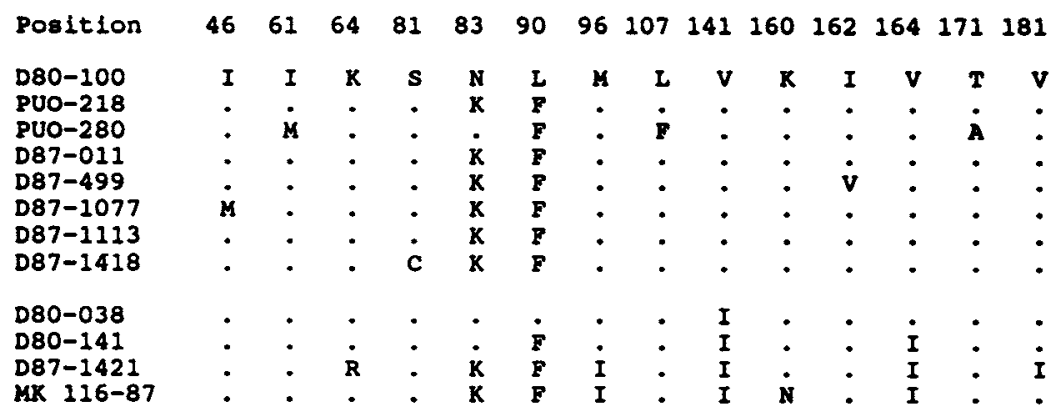

\begin{tabular}{|c|c|c|c|c|c|c|c|c|c|c|c|c|c|}
\hline Position & 190 & 203 & 308 & 345 & 346 & 359 & 366 & 447 & 468 & 478 & 484 & 491 & 149 \\
\hline D80-100 & $\mathbf{V}$ & $\mathbf{N}$ & $\mathbf{v}$ & $\mathbf{R}$ & H & $\mathbf{T}$ & $\mathbf{k}$ & A & I & $\mathbf{s}$ & I & $v$ & \\
\hline PUO- 218 & G & • & 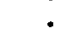 & • & - & - & N & - & $\bar{x}$ & - & - & • & \\
\hline PUO-280 & G & - & - & - & - & - & $\boldsymbol{N}$ & - & $\mathbf{M}$ & $\mathbf{P}$ & - & • & \\
\hline D87-011 & G & - & - & . & $\mathbf{Y}$ & - & $N$ & - & $\mathbf{M}$ & - & . & • & \\
\hline D87-499 & G & - & - & - & $Y$ & - & $N$ & - & $M$ & - & - & • & \\
\hline D87-1077 & G & - & - & 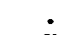 & $\mathbf{Y}$ & • & $\mathbf{N}$ & - & $M$ & - & - & • & \\
\hline D87-1113 & G & - & - & $\boldsymbol{K}$ & $\mathbf{Y}$ & - & $\mathbf{N}$ & - & $M$ & - & - & • & \\
\hline D87 & G & - & • & . & $\mathbf{Y}$ & I & $N$ & - & $\mathbf{M}$ & - & - & • & \\
\hline D $80-03$ & G & D & I & & • & 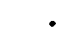 & $N$ & $\therefore$ & M & $\bullet$ & v & A & \\
\hline D80-141 & G & $D$ & $I$ & $\bullet$ & • & " & N & $\mathbf{T}$ & $\therefore$ & - & v & $A$ & \\
\hline D87-1421 & 6 & D & $I$ & . & . & : & H & - & 4 & - & v & $A$ & \\
\hline MK 116-87 & c & D & $I$ & - & $\bullet$ & $\mathbf{A}$ & $\mathbf{N}$ & . & $M$ & • & $\mathbf{v}$ & A & \\
\hline
\end{tabular}

FIGURE 1. Amino acid variation of the envelope protein among dengue type 2 viruses isolated in Thailand in 1980 and 1987 . The upper and lower panels represent selected strains of the subtypes IIIa and IIIb, respectively. Only the amino acids that differed from the D80-100 sequence are indicated for other strains. Among dengue type 2 viruses from the 1987 epidemic season, the following strains were identical in their amino acid sequence to strain D87-011: D87-167, D87-199, D87-279, D87-300, D87-642, D87-704, D87-738, D87-1121, D87-1372, D87-1441, D87-881, and DST87-60. Two other strains, D87-1040 and D87-1283, were identical to D87-499. The sequences of strains D80038, D80-100, D80-141, PUO-218, and PUO-280 were from Blok and others; ${ }^{16}$ the sequence of strain MK $116-87$ was from Duangchanda and others. ${ }^{31}$ Two other strains from Maha Sarakham were identical to MK 116-87 in the envelope protein sequence. Dot $=$ sequence identity. $\mathrm{I}=$ isoleucine; $\mathrm{K}=$ lysine; $\mathrm{S}=$ serine; $\mathrm{N}=$ asparagine; $\mathrm{L}=$ leucine; $\mathrm{M}=$ methionine; $\mathrm{V}=\mathrm{valine} ; \mathrm{T}=$ threonine; $\mathrm{F}=$ phenylalanine; $\mathrm{A}$ $=$ alanine; $C=$ cysteine; $R=$ arginine; $Q=$ glutamine $G=$ glycine; $P=$ proline; $Y=$ tyrosine; $D=$ aspartic acid.

Viral proteins in the cell lysate were resolved by SDSpolyacrylamide gel electrophoresis using the method of Laemm$\mathrm{li}^{28}$ the separating gel contained $10 \%$ acrylamide. Lysates were mixed with an equal volume of sample buffer containing 4\% SDS and $20 \%$ glycerol in Tris- $\mathrm{HCl}$ buffer, $\mathrm{pH} 6.75$, and kept at room temperature for $5 \mathrm{~min}$ before loading 10 $\mu \mathrm{l}$ per track and electrophoresing at $300 \mathrm{~V}$. The sample buffer did not include 2-mercaptoethanol. The resolved proteins were then transferred to nitrocellulose membrane in the buffer of Towbin and others ${ }^{29}$ at $500 \mathrm{~mA}$ overnight using a Transphor electrophoresis unit (Hoefer, San Francisco, CA) with cooling.

Unbound sites on the resulting blots were blocked by incubating for $30 \mathrm{~min}$ in PBS containing 5\% nonfat skim milk (PBS-SM) and probed by incubating overnight on a rocking platform with hybridoma supernatants or a pooled convalescent patient serum diluted $1: 1,000$ in PBS-SM. The blots were then washed extensively in PBS and incubated for 4 $\mathrm{hr}$ in PBS-SM containing a rabbit anti-mouse immunoglobulin conjugated with horseradish peroxidase (Dakopatts, Glostrup, Denmark) at 1:2,000 dilution or protein A conjugated with horseradish peroxidase at a concentration of 0.5 $\mu \mathrm{g} / \mathrm{ml}$. Immunoreactive bands were visualized using a colorigenic substrate containing $\mathrm{H}_{2} \mathrm{O}_{2}$ and 4-chloro-1-naphthol for $1 \mathrm{hr}$.

\section{RESULTS}

Variation of the envelope gene sequence of dengue type 2 viruses from the 1987 epidemic season. The envelope genes of 19 dengue type 2 strains from Bangkok metropolitan area were amplified and sequenced. The general structure of the envelope protein of these 1987 Bangkok isolates was identical with those previously observed in dengue type 2 prototypic strains and five 1980 Bangkok strains. ${ }^{16,24}$ Twelve conserved Cys residues and two potential N-glycosylation sites (Asn67-Thr-Thr and Asn135-Asp-Thr) were present in all strains. Variation in the deduced amino acid sequence of selected strains is shown in Figure 1.

Nucleotide sequence variations were present as single base substitutions scattered throughout the entire length of the envelope gene. The majority of substitutions occurred at the third codon position. There were no deletions nor insertions. The total number of nucleotide differences between each pairs of selected isolates is shown in Table 3. Seventeen of 19 dengue type 2 isolates differed from each other by only 16 bases or less of 1,485 bases (sequence identity of more than 98.9\%). Strains D87-642 and D87-704 were identical; they were isolated from siblings living in the same house only nine days apart. An additional strain, D87-1418, appeared to represent an extreme end in the spectrum of similar sequences since it differed from other 17 strains by up to 29 bases. In contrast to a high level of sequence identity among the first 18 strains, strain D87-1421, which was isolated from a grade IV DHF patient, varied by as many as 87-100 bases from other strains. The identity of envelope gene sequence between strain D87-1421 and other isolates ranged from $93.3 \%$ to $93.9 \%$. Strain D87-1421 appeared to be quite distinct from the rest of dengue type 2 viruses found in the 1987 epidemic year in Bangkok. 
TABLE 3

Summary of nucleotide differences (above diagonal axis) and amino acid differences (below diagonal axis) of envelope gene/protein among selected dengue type-2 viruses isolated from Thailand in 1987*

\begin{tabular}{|c|c|c|c|c|c|c|c|c|c|c|c|c|}
\hline & 1 & 6 & 11 & 12 & 13 & 15 & 17 & 18 & 20 & 21 & 22 & 23 \\
\hline 1 & - & 10 & 15 & 6 & 13 & 10 & 24 & 93 & 4 & 99 & 98 & 101 \\
\hline 6 & 1 & - & 9 & 10 & 13 & 4 & 26 & 95 & 8 & 101 & 100 & 103 \\
\hline 11 & 1 & 0 & - & 15 & 20 & 9 & 29 & 100 & 13 & 106 & 105 & 108 \\
\hline 12 & 1 & 2 & 2 & - & 13 & 10 & 22 & 91 & 4 & 95 & 94 & 97 \\
\hline 13 & 1 & 2 & 2 & 2 & - & 13 & 27 & 98 & 11 & 102 & 101 & 104 \\
\hline 15 & 1 & 0 & 0 & 2 & 2 & - & 26 & 93 & 8 & 99 & 98 & 101 \\
\hline 17 & 2 & 3 & 3 & 3 & 3 & 3 & - & 87 & 22 & 94 & 93 & 96 \\
\hline 18 & 10 & 11 & 11 & 11 & 11 & 11 & 12 & - & 9I & 16 & 16 & 15 \\
\hline 20 & 0 & 1 & 1 & 1 & 1 & 1 & 2 & 10 & - & 97 & 96 & 99 \\
\hline 21 & 10 & 11 & 11 & 11 & 11 & 11 & 11 & 4 & 10 & - & 1 & 2 \\
\hline 22 & 10 & 11 & 11 & 11 & 11 & 11 & 11 & 4 & 10 & 0 & - & 3 \\
\hline 23 & 10 & 11 & 11 & 11 & 11 & 11 & 11 & 4 & 10 & 0 & 0 & - \\
\hline
\end{tabular}

* Virus strains are indicated by study number according to Table 1 in bold.

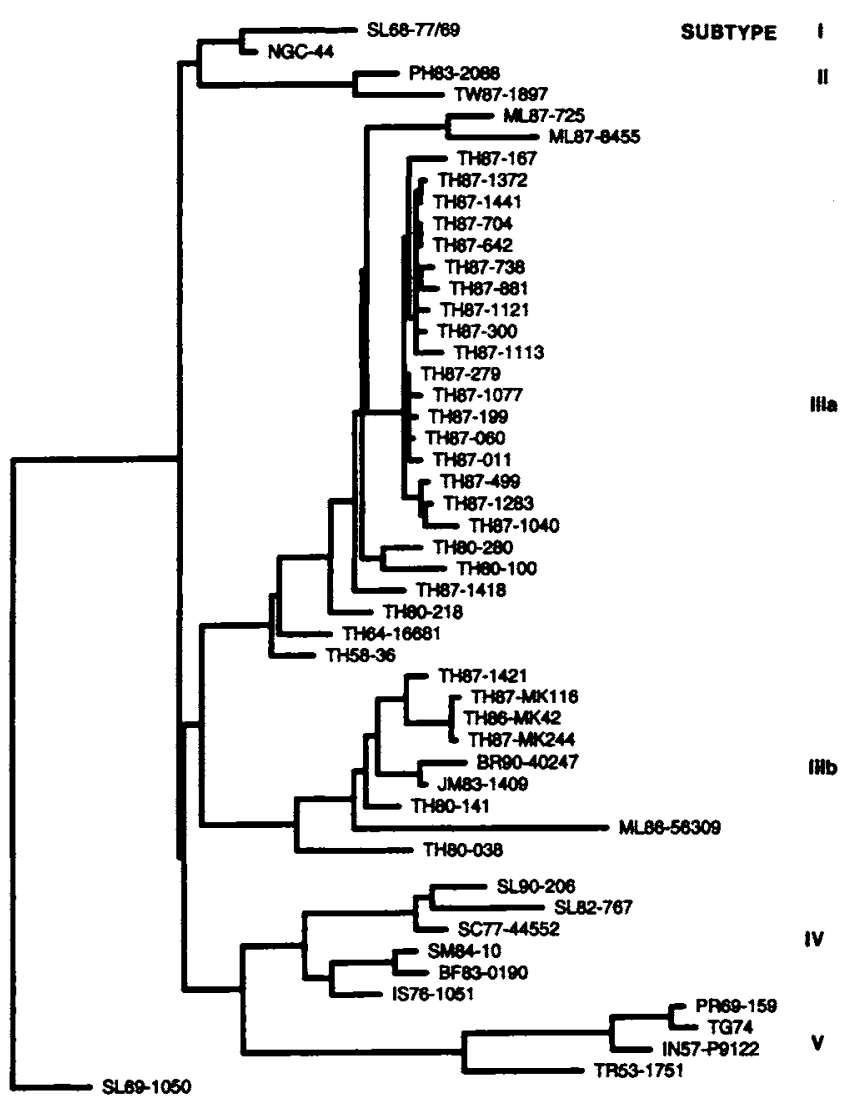

0.10

FIGURE 2. Phylogenetic relationship of dengue type 2 vinuses based on the comparison of envelope protein coding region. Twentythree dengue type 2 strains isolated during 1987 in Thailand were analyzed together with five strains from the 1980 epidemic season and selected strains from other parts of the world using the fastDNAml program. Viruses were identified by study code (Table 1) according to the country/island of origin, year of isolation, and strain designation. The bar at the bottom represents the estimated divergence under the maximum likelihood model. Sequences of viruses other than the 1987 isolates from Thailand were obtained from GenBank; all were analyzed previously by the parsimony method. ${ }^{13}$ BF = Burkina Faso; $\mathbf{B R}=$ Brazil; IN = India; IS = Indonesia; JM = Jamaica; $M L=$ Malaysia; NGC = New Guinea C; PH $=$ Philippines; PR = Puerto Rico; $S C=$ Seychelles; SL = Sri Lanka; SM $=$ Somalia; TG $=$ Tonga; TH $=$ Thailand; $T R=$ Trinidad; $T W=$ Taiwan.
At the protein level, 12 strains were identical in the amino acid sequence of the envelope protein. Three other strains (D87-499, D87-1040, and D87-1283) differed from the former strains by only one residue at the position 162 (Ile versus Val). Strains D87-1077, D87-1113, and D87-1418 each contained 1-3 additional amino acid substitutions. Among these 18 isolates, amino acid changes occurred at five positions: 46, 81, 162, 345, and 359 (Figure 1). As expected, higher level of amino acid sequence variation was seen with D87-1421, which varied from the rest by 10-12 amino acid residues (97.7-97.9\% sequence identity). Amino acid substitutions were detected at the following amino acid positions: $64,96,141,164,181,203,308,346,484$, and 491 (Figure 1). Except for the two changes that resulted in an acquisition of charged residues (position 203, Asn versus Asp; position 346, Tyr versus His), all others substitutions were conservative changes of uncharged residues (seven positions) or positively charged residues (position 64, Lys versus Arg). Thus, sequence analysis of the envelope gene and protein indicate that dengue serotype 2 viruses obtained from the 1987 epidemic season in Bangkok segregate into two groups, the major group containing 18 of 19 isolates and the minor group represented by strain D87-1421.

Comparison of the envelope gene sequence between the 1987 Bangkok isolates with other Thai strains. Direct comparison and phylogenetic analysis of the envelope sequence of dengue serotype 2 viruses obtained from 1958 to 1980 in Thailand revealed that subtype IIIa constituted the majority (five of seven strains) of all earlier Thai strains. ${ }^{13}$ The remaining two strains were classified as subtype IIIb. ${ }^{13}$ Direct comparison of all 1987 Bangkok isolates with earlier Thai strains revealed close similarity of the major group of 1987 with subtype IIIa strains (97.9-98.5\% nucleotide sequence identity). In the same manner, D87-1421 was 96.4$98.6 \%$ identical in the nucleotide sequence to the two subtype IIIb Thai strains.

Phylogenetic analysis using the maximum likelihood method confirmed close genetic relatedness between the major group and minor group of the 1987 Bangkok epidemic season with the subtype IIIa and IIIb dengue serotype 2 strains, respectively, of earlier epidemics (Figure 2). The separation was consistent in 87 and 84 of 100 bootstrap replicates analyzed. With the one exception of strain SL69-1050 
TABLE 4

Reactivities of anti-nonstructural protein 1 monoclonal antibodies against dengue type-2 viruses from Bangkok

\begin{tabular}{|c|c|c|c|c|c|c|c|c|c|c|c|}
\hline \multirow[b]{2}{*}{ Strain } & \multicolumn{10}{|c|}{ Monoclonal antibody } & \multirow[b]{2}{*}{ PCS* } \\
\hline & $\begin{array}{c}\text { MFZ/3/ } \\
\text { A3-1/F7 }\end{array}$ & $\begin{array}{c}\text { MF34/4/ } \\
\text { C4/G2 }\end{array}$ & $\begin{array}{c}\text { MF33/5/ } \\
\text { C2/E2 }\end{array}$ & $\begin{array}{c}\mathrm{BF} 1 / 3 \mathrm{H} \\
\mathrm{A} 2-1 / \mathrm{D} 11\end{array}$ & $\begin{array}{c}\mathrm{MF} 34 / 7 / \\
\mathrm{D} 2 / \mathrm{B} 2\end{array}$ & $\begin{array}{c}\text { MF34/1/ } \\
\text { A4/B2 }\end{array}$ & $\begin{array}{c}\text { MV8/1/ } \\
\text { B4-1 }\end{array}$ & $\begin{array}{l}\text { MF34/4/ } \\
\text { A5-1/DII }\end{array}$ & $\begin{array}{c}M V 3 / 3 / \\
A 6-8 / 4\end{array}$ & $\begin{array}{l}\mathrm{MV} 3 / 2 \\
\mathrm{~B} 2-2 / 2\end{array}$ & \\
\hline 16681 & + & + & + & + & + & + & + & - & + & + & + \\
\hline D87-279 & + & + & + & + & + & + & + & - & + & + & + \\
\hline D87-499 & + & + & + & + & + & + & + & - & + & + & + \\
\hline D87-642 & + & + & + & + & + & + & + & - & - & Faint & + \\
\hline D87-704 & + & + & + & + & + & + & + & - & + & + & + \\
\hline D87-1040 & + & + & + & + & + & + & + & - & + & + & + \\
\hline D87-1372 & + & + & + & + & + & + & + & - & + & + & + \\
\hline D87-1441 & + & + & + & + & + & + & + & - & + & + & + \\
\hline D87-1421 & + & + & + & + & - & - & - & + & + & Faint & + \\
\hline \multicolumn{12}{|l|}{ Mock } \\
\hline infected & - & - & - & - & - & - & - & - & - & - & - \\
\hline Pattern & 1 & 1 & 1 & 1 & 2 & 2 & 2 & 3 & 4 & 4 & \\
\hline
\end{tabular}

noted above, this analysis also confirmed the placement of other dengue type 2 isolates by the maximum parsimony method. ${ }^{13}$ The bootstrap values for inclusion within the subtypes I, II, IV, and V were $95,95,98$ and 100 , respectively.

In 1987, an additional dengue type 2 virus from Kanchanaburi province was studied (Table 1). Comparison of the envelope gene sequence of this virus and three other type 2 viruses from Maha Sarakham province in the northeastern

\section{$\begin{array}{lllllllllll}1 & 2 & 3 & 4 & 5 & 6 & 7 & 8 & 9 & 10 & 11\end{array}$}
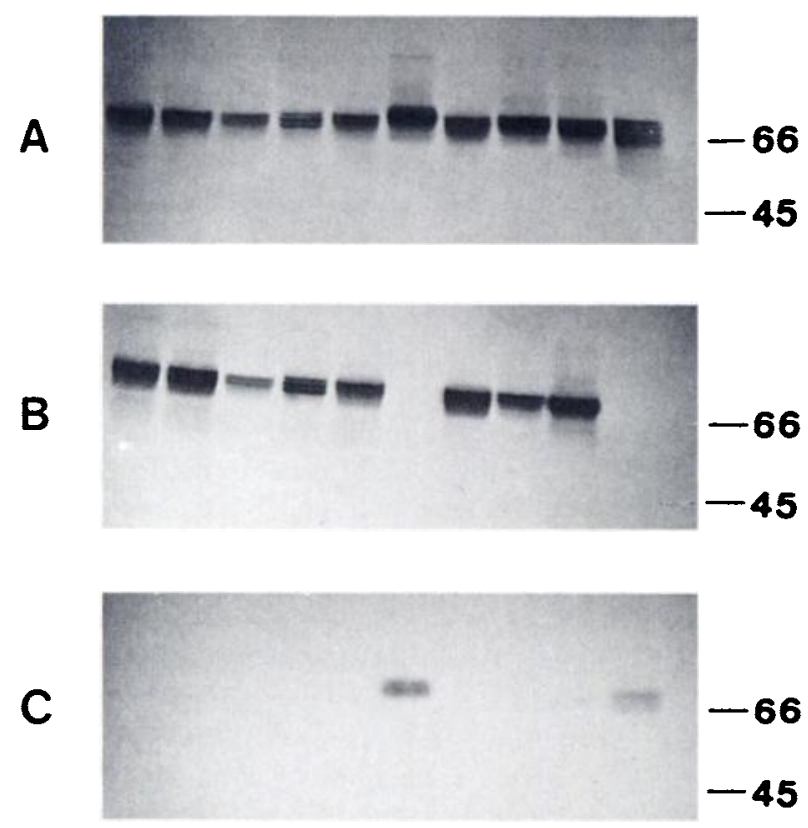

FIGURE 3. Immunoblots showing the reactivities of three antiNS1 monoclonal antibodies against various strains of dengue type 2 viruses. The samples were not reduced and not heated before running on sodium dodecyl sulfate-polyacrylamide gel. Samples were loaded as follows: lane 1, D87-279; lane 2, D87-499; lane 3, D87644; lane 4, D87-1040; lane 5, D87-1772; lane 6, D87-1421; lane 7. D87-1441: lane 8, D87-704: lane 9, strain 16681; lane 10, strain New Guinea $C$; lane 11, mock-infected control. The monoclonal antibodies used were A, MF22/3/A3-1/F7; B, MF34/7/D2/B2; C, MF34/4/A5-1/D11. The positions of molecular weight markers of $66 \mathrm{kD}$ and $45 \mathrm{kD}$ are indicated on the right. part of Thailand ${ }^{30}$ with those of Bangkok isolates revealed that all three Maha Sarakham strains were subtype IIlb viruses whereas the Kanchanaburi isolate belonged to subtype IIIa (Table 3 and Figure 2).

Comparison of the deduced envelope protein sequences of all 1987 dengue type 2 strains revealed amino acid substitutions that were characteristic of a particular strain or group of strains (Figure 1). For example, Met46, Arg64, and Ile 181 were present only in strain D87-1077 and D87-1421, respectively, whereas Lys83, Asn 160, and Ala359 occurred in the three Maha Sarakham isolates. There were five amino acid positions $(141,203,308,484$, and 491) in the envelope glycoprotein that differentiated between the subtype IIIa and IIIb dengue type 2 strains of both the 1980 and 1987 epidemic seasons in Thailand. Interestingly, the envelope glycoprotein of all 19 subtype IIIa isolates from the 1987 epidemic season (18 from Bangkok and one from Kanchanaburi province) shared a His346 $\rightarrow$ Tyr substitution (C1036 $\rightarrow U$ ), which had never been reported in any dengue type 2 viruses. Similarly, all four 1987 subtype IIIb strains (one from Bangkok and three from Maha Sarakham province) shared a unique Met96 $\rightarrow$ Ile substitution (G288 $\rightarrow$ A) (Figure 1) and two other silent base substitutions at the positions $717(\mathrm{U} \rightarrow$ C) and $765(\mathrm{C} \rightarrow \mathrm{U})$.

Immunoblot analysis of NS1 protein. Intracellular NS1 proteins derived from eight isolates from the 1987 Bangkok epidemic and two prototypic strains were analyzed by immunoblot analysis with 10 anti-dengue type 2 NSI monoclonal antibodies. Under the nonreducing running conditions used, NSI dimer remained intact and was recognized by high-titered human serum pooled from convalescent dengueinfected patients, which were used as a positive control. Four reactivity patterns were detected (Table 4 ). In the first pattern, four antibodies (MF2/3/A3-1/F7, MF34/4/C4/G2, $\mathrm{MF} 33 / 5 / \mathrm{C} 2 / \mathrm{E} 2$, and $\mathrm{BF} 1 / 3 / \mathrm{A} 2-1 / \mathrm{D} 11)$ reacted with all dengue type 2 isolates tested (Figure $3 \mathrm{~A}$ ). The second and third patterns were reciprocal in that MF34/7/D2/B2, MF34/1/A4/B2, and MV8/1/B4-1 reacted with eight of eight subtype IIIa strains but not with D87-1421 (subtype IIIb) and New Guinea C (subtype I) whereas MF34/4/A5-1/D1 1 reacted only with D87-1421 and New Guinea C (Figures 3B and $\mathrm{C}$ ). The second pattern appeared to reflect an epitope 
that was common among the subtype IIla viruses. In contrast, the third pattern was indicative of the epitope found in other subtypes but absent from subtype IIIa viruses. In the fourth pattern, the antibodies MV3/3/A6-8/4 and MV3/2/B2$2 / 2$ recognized epitopes that were differentially absent in individual subtype IIIa strains. Based on these reactivities, the subtype IIIa strains can be separated from the other two subtypes by using the second and third groups of anti-NS1 monoclonal antibodies.

\section{DISCUSSION}

The 1987 season was the largest dengue epidemic ever recorded in Thailand. ${ }^{70}$ In Bangkok, dengue type 1,2 , and 3 viruses were recovered. ${ }^{9}$ Among serotype 2 viruses, the majority (18 of 19) were quite homogeneous and corresponded to the subtype IIIa of the five-subtype phylogenetic classification. ${ }^{13}$ Subtype IIIb viruses were rare; strain D871421 was the only subtype IIIb detected. Similar to previous reports, ${ }^{16-18}$ there was no correlation between either specific envelope gene variant or virus subtype with clinical severity.

In addition to strain D87-1421, there were only two other

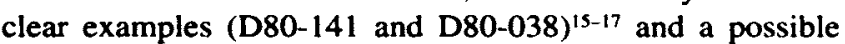
member $(516)^{12.13}$ of subtype IIIb viruses identified in Bangkok. Although a reliable estimate of the distribution of the subtype IIIb strain during the 1980-1987 epidemic seasons in Bangkok was not available, it was likely that subtype IIIb constituted a minority among dengue type 2 viruses present in this city. In a longitudinal study of dengue type 2 viruses isolated during the period 1975-1986, not a single subtype IIIb virus was detected among 26 isolates from Bangkok when at most 4-5 isolates were analyzed for each year. ${ }^{14}$ Serotype IIIb vinuses were detected only in years when large numbers of isolates were examined: 10 in $1980^{15}$ and 19 in 1987. In contrast, the finding of Duangchanda and others ${ }^{31}$ suggested the predominance of subtype IIIb viruses in Maha Sarakham province during the same period. This discrepancy raised a possibility that similar to the well known variation in serotype distribution, there existed an uneven distribution of dengue subtypes in various localities in each epidemic season. This possibility has important implications for the efficacy testing of dengue type 2 vaccine candidate in the near future; it should be confirmed by using a larger number of isolates derived concurrently from several localities.

Comparison of the envelope gene sequence of the subtype IIIa and IIIb strains isolated in 1987 with those of 1980 revealed in both groups three types of substitutions. The first type was represented by substitutions that were unique to an individual isolate or group of isolates. These changes might result from the most recent random mutational events causing the generation of new strains (genetic drift). The second type of substitutions reflected amino acid positions that were different between subtype IIIa and IIIb viruses of both the 1980 and 1987 epidemic seasons. These were mainly conservative amino acid changes that had been fixed in both subtypes at least since 1980 . The third type constituted, respectively, an amino acid change and an amino acid change plus two silent nucleotide substitutions, which were shared by all subtype IIIa and subtype IIIb viruses of 1987 but were absent from corresponding subtypes of 1980 . It was unlikely that the last finding was due to geographic clustering because viruses were derived from patients living in several districts and adjacent provinces of the Bangkok metropolitan area in addition to provinces located far from Bangkok. Likewise, selection bias with regard to time could be rejected since virus strains were isolated more or less evenly throughout the year. The third type of variation may possibly be derived from random mutation if there existed very strong selective pressure for maintaining these changes in viral populations. It was difficult, however, to envision a similar or identical biological advantage of the new substitution at two distinct positions (\#96 versus \#346) that localized on separate structural domains of flaviviral envelope glycoprotein. ${ }^{32}$ Alternatively, it was more likely that the third type of substitutions reflected a genetic bottleneck occurring between 1980 and 1986, which allowed continued transmission of one (or only a few) precursor strains, most likely that of subtype IIIa, into 1987. The finding was also compatible with extinction and recent introduction from single source. During the 1986-1987 epidemic seasons, the subtype IIIa and subtype IIIb dengue viruses had also been identified in Malaysia. ${ }^{13 .}{ }^{33}$ However, it is unlikely that they could serve as single sources of reintroduction into Bangkok, if extinction did occur, because the Malaysian subtype IIIa and IIIb strains lacked the unique Tyr346 and 11 e96 residues common in the corresponding Thai strains. Based on the finding that subtype IIIa and IIIb strains isolated from provinces outside Bangkok were very similar to those found in Bangkok, extinction of dengue type 2 viruses in Bangkok followed by introduction from other sources in Thailand would have the same effect as a genetic bottleneck occurring within Bangkok. To our knowledge, this may represent the first example of a genetic bottleneck detected among dengue type 2 viruses in nature.

Genetic bottleneck and extinction are thought to play important roles in the evolution of mosquito-borne viruses. ${ }^{34}$ Due to the complexity of interactions between viruses with two distinct hosts (mosquito and human) required to maintain transmission, extinction at the local level might occur frequently. ${ }^{34}$ Indeed, extinction of one or multiple dengue serotypes has been documented.9.35 On the contrary, demonstration of a genetic bottleneck in virus populations has been difficult because this required comparisons of the nucleotide sequences of a large number of sequential samples. Until now, clear examples of a genetic bottleneck were known at the level of infected individuals, especially following the infection with human immunodeficiency virus (HIV) and hepatitis $\mathrm{C}$ virus. ${ }^{36}$ The significance of a genetic bottleneck occurring in vivo at both the individual and population levels is not yet clear. Based on in vitro experiments, a genetic bottleneck resulted in a dramatic reduction in the fitness of RNA viruses due to the greater chance of involvement of less-fit, but more numerous, variants in the viral population. ${ }^{36-38}$ As shown in one example, once fitness reduction had occurred as a result of a bottleneck, even a very high level of subsequent amplification might not be able to restore replicative fitness to its original status. ${ }^{39}$ A recent description of an HIV-infected long-term nonprogressor, who harbored the nef-deleted variant with low level of virus replication, may well represent an in vivo example of a genetic bottleneck with a subsequent fitness decrease at the individual level, although the original source of infection was not characterized. ${ }^{40}$ 
Gradual change in the genome of dengue viruses observed in nature is likely to be due to the interaction of genetic alteration and structural and/or functional constraints associated with back and forth passages between two hosts and immune selection in the human host. Genetic alteration at the individual and population levels may possibly include random point mutation, genetic recombination, bottleneck, and local extinction and reintroduction. ${ }^{34}$ The frequency of which these multiple types of changes occur in nature is unknown and may vary from place to place. In several cases of concurrent infection with two dengue serotypes, no evidence for genetic recombination was observed, indicating that recombination may be a rare event for dengue viruses. ${ }^{41.42}$ Currently, how genetic changes contribute to the transmission and spread of dengue viruses and their impact on the clinical severity in an endemic area such as Thailand are unknown. These will require careful monitoring of dynamic changes that occur in the virus population and their hosts.

When the subtype IIIa and IIIb viruses from the 1987 Bangkok epidemic were analyzed with anti-NS1 antibodies, two reciprocal reactivity patterns correlated with the subtyping based on envelope glycoprotein variation, most evidently for subtype IIIa strains. This finding was in good agreement with a previous comparison of dengue viruses isolated with the 1980 epidemic, which revealed that the subtype IIIa and IIIb viruses also segregated when the NS1 sequences were compared. ${ }^{17}$ Our finding provided further structural evidence in support of the genetic subtyping of dengue serotype 2 viruses based on primary sequence variation. Because no other subtypes of dengue type 2 viruses were found in Thailand and Malaysia, ${ }^{13},{ }^{33}$ the anti-NS1 monoclonal antibodies reported herein should be useful as an additional tool for the identification of the subtypes IIIa and IIIb in this setting, but they may not be sufficient to differentiate other subtypes. Clones with specificity for the subtypes I, II, IIIb, IV, and V will be needed before these antibodies can be used in other geographic areas.

Acknowledgments: We thank Drs. Bruce Innis and Ananda Nisalak (Department of Virology, Armed Forces Research Institute of Medical Sciences, Bangkok) for supplying dengue viruses and reagents; Dennis Trent (Centers for Disease Control and Prevention, Fort Collins, $\mathrm{CO}$ ) for gifts of reagents and encouragement: Prida Malasit (Siriraj Medical School, Bangkok); Toshihiko Fukunaga and Yoshihiro Makino (University of the Ryukyus, Nishihara, Okinawa) for providing cells and an assay protocol; and Siraporn Swasdivorn (Maha Sarakham Hospital, Maha Sarakham) for providing information on patients.

Financial support: This research was supported by grant no. 9.322 , Program in Science and Technology Cooperation, Office of the Science Advisor, U. S. Agency for International Development, and a grant from the International Development Research Center (IDRC) to Mary Jane Cardosa.

Authors' addresses: Nopporn Sittisombut, Anchalee Sistayanarain, Supaporn Damrongdachakul, Jiraporn Supawadee, and Niwat Maneekarn, Department of Microbiology, Faculty of Medicine, Chiang Mai University, 110 Intavaroros Street, Chiang Mai 50200, Thailand. Mary Jane Cardosa, School of Pharmaceutical Sciences, Universiti Sains Malaysia, Penang 11800, Malaysia. Mika Salminen, Henry M. Jackson Foundation, Rockville, MD 20850. Siripen Kalayanarooj, Department of Pediatrics, Children's Hospital, Ministry of Public Health, Bangkok 10400, Thailand. Suntharee Rojanasu- phot, Virus Research Institute, Department of Medical Sciences, Ministry of Public Health, Nonthaburi 11000, Thailand.

\section{REFERENCES}

1. Halstead SB, 1980. Immunological parameters of togavirus disease syndromes. Schlesinger RW, ed. Togaviruses: Biology, Structure, Replication. New York: Academic Press, 107-173.

2. Monath TP, 1986. Pathobiology of the flavivinuses. Schlesinger $\mathrm{S}$, Schlesinger MJ, eds. The Togaviridae and Flaviviridae. New York: Plenum Press, 375-440.

3. Halstead SB, 1988. Pathogenesis of dengue: challenges to molecular biology. Science 239: 476-481.

4. Henchal EA, Putnak JR, 1990. The dengue viruses. Clin Microbiol Rev 3: 376-396.

5. Monath TP, 1994. Dengue: the risk to developed and developing countries. Proc Natl Acad Sci USA 91: 2395-2400.

6. Udomsakdi $S, 1973$. Studies on hemorrhagic fever in Thailand 1958-1971: a review. J Med Assoc Thailand 56: 40-66.

7. Nimmanitya $S, 1987$. Dengue hemorrhagic fever in Thailand. Southeast Asian J Trop Med Public Health 18: 291-294.

8. Burke DS, Nisalak A, Johnson DE, McN. Scott R, 1989. A prospective study of dengue infection in Bangkok. Am J Trop Med Hyg 38: 172-180.

9. Nisalak A, Nimmanitya S, Innis B, 1990. Severe dengue infections among children in metropolitan Bangkok, 1973-1989: description of epidemics and characterization of virus serotypes associated with them. Southeast Asian J Trop Med Hyg Public Health 21: 678-679.

10. Trent DW, Grant JA, Rosen L, Monath TP, 1983. Genetic variation among dengue 2 viruses of different geographic origin. Virology 128: 271-284.

11. Monath TP, Wands JR, Hill LJ, Brown NV, Marciniak RA, Wong MA, Gentry MK, Burke DS, Grant JA, Trent DW, 1986. Geographic classification of dengue- 2 virus strains by antigen signature analysis. Virology 154: 313-324.

12. Rico-Hesse $R, 1990$. Molecular evolution and distribution of dengue viruses type 1 and 2 in nature. Virology 174: 479493.

13. Lewis JA, Chang GJ, Lanciotti RS, Kinney RM, Mayer LW, Trent DW, 1993. Phylogenetic relationships of dengue-2 viruses. Virology 197: 216-224.

14. Trent DW, Grant JA, Monath TP, Manske CL, Corina M, Fox GE, 1989. Genetic variation and microevolution of dengue 2 virus in Southeast Asia. Virology 175: 523-535.

15. Walker PJ, Henchal EA, Blok J, Repik PM, Henchal LS, Burke DS, Robbins SJ, Gorman BM, 1988. Variation in dengue type 2 viruses isolated in Bangkok during 1980. J Gen Virol 69: 591-602.

16. Blok J, Samuel S, Gibbs AJ, Vitaratana UT, 1989. Variation of the nucleotide and encoded amino acid sequences of the envelope gene from eight dengue-2 viruses. Arch Virol 105: 39-53.

17. Blok J, Gibbs N, McWilliam SM, Vitaratana UT, 1991. NS1 gene sequences from eight dengue-2 viruses and their evolutionary relationships with other dengue-2 viruses. Arch Virol 118: 209-223.

18. Chungue E, Deubel V, Cassar O, Laille M, Martin PMV, 1993. Molecular epidemiology of dengue 3 viruses and genetic relatedness among dengue 3 strains isolated from patients with mild or severe form of dengue fever in French Polynesia. $J$ Gen Virol 74: 2765-2770.

19. World Health Organization, 1986. Dengue Haemorrhagic Fever: Diagnosis, Treatment and Control. Geneva: World Health Organization, 1-58.

20. Henchal EA, Gentry MK, McCown JM, Brandt WE, 1982. Dengue virus-specific and flavivirus group determinants identified with monoclonal antibodies by indirect immunofluorescence. Am J Trop Med Hyg 31: 830-836.

21. Okuno Y, Sasao F, Fukunaga T, Fukai K, 1977. An application of PAP (peroxidase-anti-peroxidase) staining technique for the rapid titration of dengue virus type 4 infectivity. Biken $J 20$ : 29-33. 
22. Ishimine $T$, Tadano $M$, Fukunaga $T$, Okuno $Y, 1987$. An improved micromethod for infectivity assays and neutralization tests of dengue viruses. Biken $J$ 30: 39-44.

23. Halstead SB, Simasthien P, 1970. Observations related to the pathogenesis of dengue hemorrhagic fever. II. Antigenic and biologic properties of dengue viruses and their association with disease response in the host. Yale J Biol Med 42: 276292.

24. Blok J, McWilliam SM, Butler HC, Gibbs N, Weiller G, Herring BL, Hemsley AC, Aaskov JG, Yoksan S, Bhamarapravati N, 1992. Comparison of a dengue-2 virus and its candidate vaccine derivative: sequence relationships with the flaviviruses and other viruses. Virology 187: 573-590.

25. Felsenstein J, 1991. PHYLIP Manual. Version 3.4. Berkeley, CA: University Herbarium, University of California.

26. Kohler G, Milstein C, 1975 . Continuous cultures of fused cells secreting antibody of predefined specificity. Nature 256: 495497.

27. Cardosa MJ, Tio PH, 1991. Dot enzyme immunoassay: an alternative diagnostic aid for dengue fever and dengue haemorrhagic fever. Bull World Health Organ 69: 741-745.

28. Laemmli UK, 1970. Cleavage of structural proteins during the assembly of the head of bacteriophage T4. Nature 227: 680685.

29. Towbin H, Staehelin T, Gordon J, 1979. Electrophoretic transfer of proteins from polyacrylamide gels to nitrocellulose sheets: procedure and some applications. Proc Natl Acad Sci USA 76: $4350-4354$

30. Halstead SB, 1992. The XXth century dengue epidemic: need for surveillance and research. World Health Stat $Q 45$ : 292298.

31. Duangchanda S, Tanaka M, Morita K, Rojanasuphot S, Igarashi A, 1994. Comparative nucleotide and deduced amino acid sequence of the envelope glycoprotein gene among three dengue virus type 2 strains isolated from patients with different disease severities in Maha Sarakham, northeast Thailand. Southeast Asian J Trop Med Public Health 25: 243-251.

32. Rey FA, Heinz FX, Mandl C, Kunz C, Harrison SC, 1995. The envelope glycoprotein from tick-borne encephalitis virus at 2 $A^{\circ}$ resolution. Nature 375: 291-298.
33. Samuel S, Koh CL, Blok J, Pang T, Lam SK, 1989. Nucleotide and encoded amino acid sequences of the envelope protein gene of a Malaysian dengue-2 virus isolated from a patient with dengue fever or DHF or DS5 (three separate reports). Nucleic Acids Res 17: 8875, 8887, and 8888 .

34. Scott TW, Weaver SC, Mallampalli VL, 1994. Evolution of mosquito-borne viruses. Morse SS, ed. The Evolutionary Biology of Viruses. New York: Raven Press, 293-324.

35. Winter PE, Nantapanich S, Nisalak A, Udomsakdi S, Dewey RW, Russell DK, 1969. Recurrence of epidemic dengue hemorrhagic fever in an insular setting. Am J Trop Med Hyg 18 . 573-579.

36. Duarte EA, Novella IS, Ledesma S, Clarke DK, Moya A, Elena SF, Domingo E, Holland JJ, 1994. Subclonal components of consensus fitness in an RNA virus clone. $J$ Virol 68: 42954301.

37. Clarke DK, Duarte EA, Moya A, Elena SF, Domingo E, Holland $\mathrm{J}, 1993$. Genetic bottlenecks and population passages cause profound fitness differences in RNA viruses. $J$ Virol 67: 222228.

38. Chao L, 1990. Fitness of RNA virus decreased by Muller's ratchet. Nature 348: 454-455.

39. Duarte EA, Clarke DK, Moya A, Elena SF, Domingo E, Holland JJ, 1993. Many-trillionfold amplification of single RNA virus particles fails to overcome the Muller's ratchet effect. $J$ Virol 67: 3620-3623.

40. Deacon NJ, Tsykin A, Solomon A, Smith K, Ludford-Menting M, Hooker DJ, McPhee DA, Greenway AL, Ellett A, Chatfield C Lawson VA, Crowe S, Maerz A, Sonza S, Learmont J, Sullivan JS, Cunningham A, Dwyer D, Dowton D, Mills J, 1995. Genomic structure of an attenuated quasi species of HIV-1 from a blood transfusion donor and recipients. Science 270: 988-991.

41. Laille M, Deubel V, Sainte-Marie FF, 1991. Demonstration of concurrent dengue 1 and dengue 3 infection in six patients by the polymerase chain reaction. $J$ Med Virol 34: 51-54.

42. Maneekarn M, Morita K, Tanaka M, Igarashi A, Usawattanakul W, Sirisanthana V, Innis BL, Sittisombut N, Nisalak A, Nimmanitya S, 1993. Application of polymerase chain reaction for identification of dengue viruses isolated from patient sera. Microbiol Immunol 37: 41-47. 